\title{
RELIABILITY AND AVAILABILITY ANALYSIS OF SOME SYSTEMS WITH COMMON-CAUSE FAILURES USING SPICE CIRCUIT SIMULATION PROGRAM
}

\author{
MUHAMMAD TAHER ABUELMA'ATTI ${ }^{\mathrm{a}, *}$ \\ and ISA SALMAN QAMBER ${ }^{b}$ \\ ${ }^{a}$ King Fahd University of Petroleum and Minerals, \\ Box 203 Dhahran 31261, Saudi Arabia; \\ ${ }^{\mathrm{b}}$ University of Bahrain, P.O. Box 32038 Isa Town Bahrain
}

(Received 31 August 1998; In final form 3 December 1998)

\begin{abstract}
The effectiveness of SPICE circuit simulation program in calculating probabilities, reliability, steady-state availability and mean-time to failure of repairable systems described by Markov models is demonstrated. Two examples are presented. The first example is a warm standby system with common-cause failures and human errors. The second example is a non-identical unit parallel system with common-cause failures. In both cases recourse to numerical solution is inevitable to obtain the Laplace transforms of the probabilities. Results obtained using SPICE are compared with previously published results obtained using the Laplace transform method. Full SPICE listings are included.
\end{abstract}

Keywords: Reliability; availability; SPICE simulation

\section{INTRODUCTION}

In the design, development, tuning and upgrading of engineering systems, performance and reliability are two of the main factors which must be taken into consideration. Whether it is for predicting the behavior of new designs or studying possible changes to existing ones, models of such systems are essential tools for such investigations. Several

\footnotetext{
*Corresponding author.
} 
mathematical evaluation techniques can be used to analyze these models. Of particular interest here is the Markov analysis technique which is widely used for calculating the reliability and availability of repairable systems.

According to the state transition diagram, the state transition equation of a repairable electrical system, with $N$ state probabilities, can be represented by $N$ equations of the form

$\frac{d P_{n}(t)}{d t}=a_{1 n} P_{1}(t)+\cdots-a_{n n} P_{n}(t)+a_{N n} P_{N}(t), \quad n=1,2, \ldots, N$,

where $\left(d P_{n}(t) / d t\right)$ represents the differentiation of the $n$ th-state probability $P_{n}(t)$,

$$
\begin{aligned}
a_{11}= & a_{12}+a_{13}+\cdots+a_{1 N} \\
& \cdots \cdots+\cdots+\cdots \\
a_{n n}= & a_{n 1}+a_{n 2}+\cdots+a_{n N}
\end{aligned}
$$

and

$$
a_{N N}=a_{N 1}+a_{N 2}+\cdots+a_{N(N-1)}
$$

where $N$ is the number of states. Eq. $(1 \mathrm{n}), n=1,2, \ldots, N$, can be solved using the Laplace transform method [1 $1-5]$ yielding expressions for the probability, $P_{n}(t)$, that the overall system under consideration is in state $n$ at time $t$ for $n=1,2, \ldots, N$. Alternatively, the steady state probabilities, $P_{n}, n=1,2, \ldots, N$ can be obtained by setting the derivatives with respect to time, of equations $(1 \mathrm{a})-(1 \mathrm{~N})$, equal to zero and using the relationship

$$
\sum_{n=1}^{N} P_{n}(t)=1
$$

to solve the resulting $N$ algebraic equations [6,7]. While these approaches are attractive for relatively simple systems, it is virtually impossible to obtain a general time dependent expressions for the probabilities of more complicated systems. Moreover, in some cases recourse to numerical methods may be inevitable in order to solve high 
order algebraic equations before obtaining the Laplace transforms of the different probabilities [1].

On the other hand, the SPICE circuit simulation program is a generalpurpose program which can be used for d.c., transient and a.c. analysis. Although it was initially developed for integrated circuit analysis, it is now widely used for many non-integrated circuit applications. Recently, the use of SPICE capabilities in studying the behavior of multi-state systems described by Markov models was investigated [8]. The equivalent circuits used and the results obtained for the steady-state probabilities of four-state and five-state models prove that SPICE circuit simulation program is a useful tool for studying the behavior of multistate systems described by the Markov models.

The major intention of this paper is to present equivalent circuits for studying the behavior of two multi-state systems described by the Markov models. The first system is a non-identical unit parallel system with common-cause failures [5]. The second system is warm standby system with common-cause failures and human errors [1]. The first system requires the algebraic solution of a fourth-order polynomial before obtaining the Laplace transforms of the different probabilities and the second system requires numerical solution of a fifth-order polynomial before obtaining the Laplace transforms of the different probabilities. Using the proposed equivalent circuits, reliability and availability analysis of these systems can be easily performed using SPICE. From the SPICE output file the following can be obtained:

1. The probability, $P_{n}(t)$, that the system is in state $n$ at time $t$.

2. The system reliability, $R_{s}(t)$, that is the probability that the system is in an up state.

3. The system steady state availability, $A V_{s s}$, that is the long term probability that the system is in an up state.

4. The system mean time to failure, MTTF.

\section{PROPOSED MODEL}

According to Ref. [7], an appropriate source for the model must be identified and this source-state is recommended to be the equation having the larger number of terms. The source equation is replaced by Eq. (2). It is worth mentioning that, the choice of a particular equation 
as the source is not critical and any of the state Eq. (1n) can be assigned as the source-state. After replacing the selected source equation by Eq. (2), performing the necessary modifications and rearrangements, Eq. (1n), $n=1,2, \ldots, N$ can be rewritten as

$$
P_{s}(t)=1-\sum_{m=1, m \neq s}^{N} P_{m}(t)
$$

and

$$
P_{n}(t)=\sum_{m=1, m \neq n}^{N} \alpha_{n m} P_{m}(t)+\beta_{n} \frac{d P_{n}(t)}{d t}, \quad n=1, \ldots, N, n \neq s
$$

The electrical equivalent circuit of Eq. (3) is shown in Figure 1. In the circuit, the current is the analog of $P_{n}(t)$, the voltage across the inductor is the analog of the derivative with respect to time, $\left(d P_{n}(t) / d t\right)$, the voltage across the capacitor is the analog of the integration with respect to time, $\int_{0}^{t} P_{n}(t) d t$, and the voltage-controlled current sources (VCCSs), $G_{1}, G_{2}, \ldots, G_{N}$, represent the right-hand sides of Eq. (3). The resistors, $R$, in parallel with the capacitors prevent floating nodes to satisfy SPICE rules. These resistors are too large to avoid any significant effect on the circuit dynamics. The integrations $\int_{0}^{t} P_{n}(t) d t$, $n=1,2, \ldots, N, n \neq s$ are required to calculate the VCCSs, $G_{n}, n=$ $1,2, \ldots, N, n \neq s$, when Eq. ( $3 \mathrm{n})$ is in the form

$$
\frac{d P_{n}(t)}{d t}=\sum_{m=1, m \neq n}^{N} \gamma_{n m} P_{m}(t), \quad n=1, \ldots, N, n \neq s
$$

In such case (or cases), the probability $P_{n}(t)$ can be expressed as

$$
P_{n}(t)=\int_{0}^{t} \sum_{m=1, m \neq n}^{N} \gamma_{n m} P_{m}(t) d t, \quad n=1, \ldots, N, n \neq s
$$

The polynomial feature of SPICE will be used to represent these VCCSs [8]. In the SPICE input file of the circuit of Figure 1, the initial conditions of $P_{n}(t), n=1,2, \ldots, N$ are realized by the IC option, the transient analysis option TRAN will be used to perform the transient 


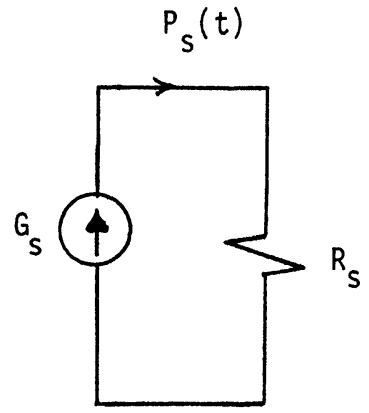

(a)

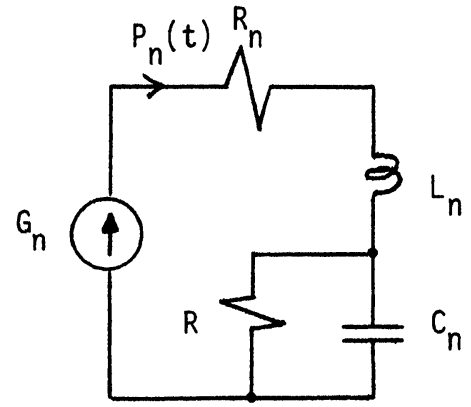

(b)

FIGURE 1 Equivalent circuits of: (a) Eq. (3a) and (b) Eq. (3n) $R_{s}=R_{n}=1 \Omega$, $L_{n}=1 \mathrm{H}, C_{n}=1 \mathrm{~F}, R=1 \mathrm{G} \Omega, n=1,2, \ldots, N, n \neq s$.

analysis of the circuit of Figure 1 and the SPICE output file will contain the probabilities $P_{n}(t), n=1,2, \ldots, N$ as a function of time.

Using the probabilities $P_{n}(t), n=1,2, \ldots, N$, the system reliability can be calculated using Eq. (6).

$$
R_{s}(t)=\sum_{n=1}^{M} P_{n}(t)
$$

where $M<N$ is the number of states with the system up. The electrical equivalent circuit of Eq. (6) is shown in Figure 2. In the circuit the current is the analog of the system reliability $R_{s}(t)$ and the VCCS, $G_{R}$, represents the right hand-side of Eq. (6).

The steady-state availability, defined as the long term probability that the system is in the up state, can be expressed as

$$
A V_{s s}=\sum_{n=1}^{M} P_{n}(\infty)
$$

where $M<N$ is the number of states with the system up. Thus, the steady-state system availability can be obtained directly from the SPICE output file. 


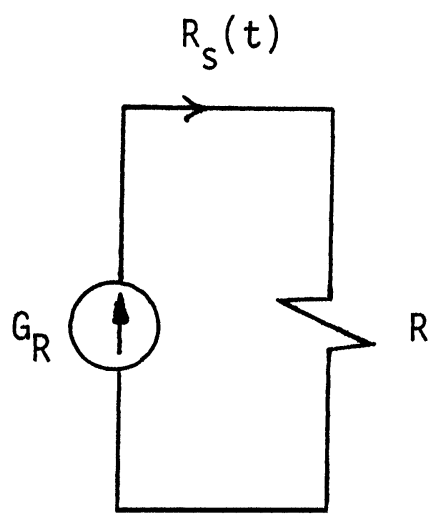

FIGURE 2 Equivalent circuit of Eq. (6) $R=1 \Omega$.

The system mean-time to failure can be expressed as

$$
\operatorname{MTTF}=\int_{0}^{\infty} R_{s}(t) d t
$$

The electrical equivalent circuit of Eq. (6b) is shown in Figure 3. In the circuit the voltage across the capacitor is the analog of the system MTTF.

\section{EXAMPLES}

The circuits of Figures $1-3$ can be used for obtaining the probabilities $P_{n}(t), n=1,2, \ldots, N$, the reliability $R_{s}(t)$, the steady-state availability $A V_{s s}$ and the MTTF of any system described by a set of state transition equations. Of particular interest here are systems for which recourse to numerical solution of high order polynomials is inevitable.

Example 1 A non-identical unit parallel system with common-cause failures [5].

Figure 4 shows the state transition diagram along with the corresponding failure rates and repair rates of the non-identical unit parallel system with common-cause failures [5]. The state transition equations 


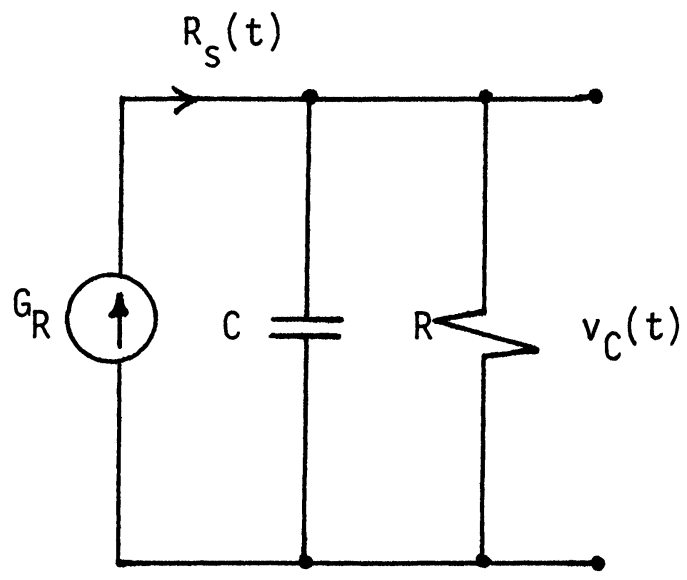

FIGURE 3 Equivalent circuit of Eq. (6b). $R=1 \mathrm{G} \Omega, C=1 \mathrm{~F}, \mathrm{MTTF}=V_{c}(t)$.

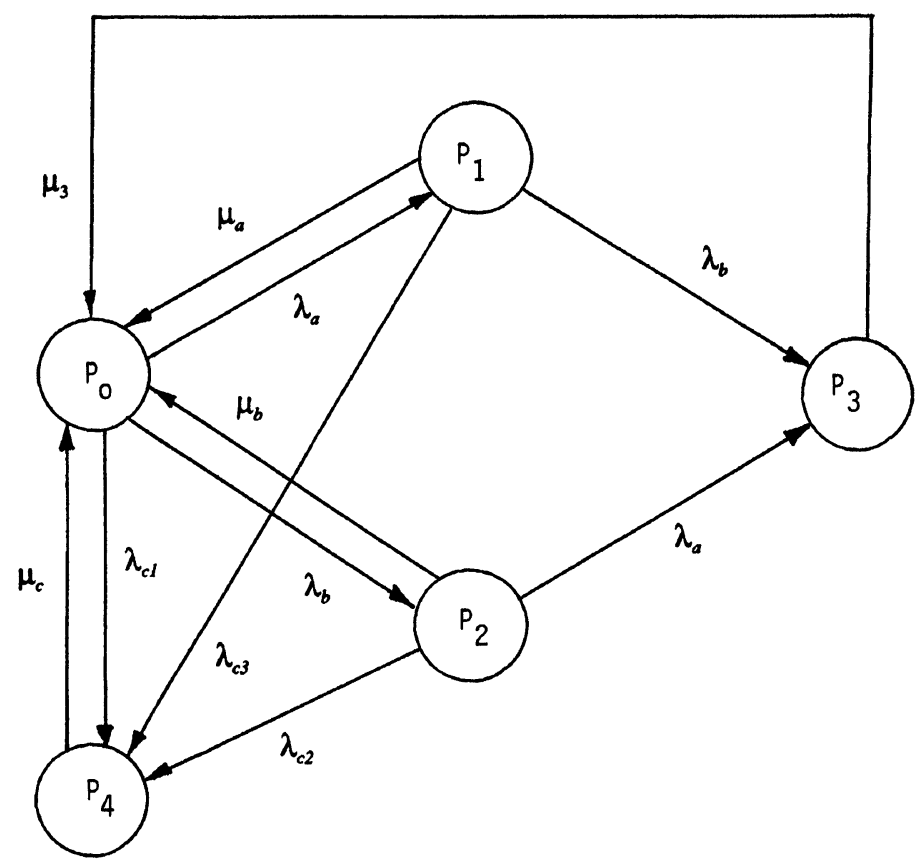

FIGURE 4 State transition diagram of Example 1 [5] State 0: Both units operating, State 1: Unit A failed, unit B working, State 2: Unit B failed, unit A working, State 3: Both units failed, State 4: System failed due to common cause failures. 
of this system can be written as:

$$
\begin{aligned}
\frac{d P_{0}(t)}{d t}= & -\left(\lambda_{a}+\lambda_{b}+\lambda_{c 1}\right) P_{0}(t)+\mu_{a} P_{1}(t) \\
& +\mu_{b} P_{2}(t)+\mu_{3} P_{3}(t)+\mu_{c} P_{4}(t) \\
\frac{d P_{1}(t)}{d t}= & \lambda_{a} P_{0}(t)-\left(\mu_{a}+\lambda_{b}+\lambda_{c 3}\right) P_{1}(t) \\
\frac{d P_{2}(t)}{d t}= & \lambda_{b} P_{0}(t)-\left(\mu_{b}+\lambda_{a}+\lambda_{c 2}\right) P_{2}(t) \\
\frac{d P_{3}(t)}{d t}= & \lambda_{b} P_{1}(t)+\lambda_{a} P_{2}(t)-\mu_{3} P_{3}(t)
\end{aligned}
$$

and

$$
\frac{d P_{4}(t)}{d t}=\lambda_{c 1} P_{0}(t)+\lambda_{c 3} P_{1}(t)+\lambda_{c 2} P_{2}(t)-\mu_{c} P_{4}(t)
$$

Solving Eqs. (7a-7e) with the aid of Laplace transforms yields the state probabilities in terms of the real roots of a fourth-order polynomial. However, obtaining the real roots of a fourth-order polynomial is deeply involved in a sea of algebra [5].

Following the procedure described in the previous section, assuming that Eq. (7a) is the source equation, Eqs. $(7 a-7 e)$ can be rewritten as

$$
\begin{gathered}
P_{0}(t)=1-P_{1}(t)-P_{2}(t)-P_{3}(t)-P_{4}(t) \\
P_{1}(t)=\alpha_{1}\left(1-P_{2}(t)-P_{3}(t)-P_{4}(t)-\frac{1}{\lambda_{a}} \frac{d P_{1}(t)}{d t}\right) \\
P_{2}(t)=\alpha_{2}\left(1-P_{1}(t)-P_{3}(t)-P_{4}(t)-\frac{1}{\lambda_{b}} \frac{d P_{2}(t)}{d t}\right) \\
P_{3}(t)=\alpha_{3}\left(\lambda_{b} P_{1}(t)+\lambda_{a} P_{2}(t)-\frac{d P_{3}(t)}{d t}\right)
\end{gathered}
$$


and

$$
P_{4}(t)=\alpha_{4}\left(\frac{\lambda_{c 3}-\lambda_{c 1}}{\lambda_{c 1}} P_{1}(t)+\frac{\lambda_{c 2}-\lambda_{c 1}}{\lambda_{c 1}} P_{2}(t)-P_{3}(t)-\frac{1}{\lambda_{c 1}} \frac{d P_{4}(t)}{d t}\right)
$$

where

$$
\begin{gathered}
\alpha_{1}=\frac{\lambda_{a}}{\lambda_{a}+\lambda_{b}+\lambda_{c 3}+\mu_{a}} \\
\alpha_{2}=\frac{\lambda_{b}}{\lambda_{a}+\lambda_{b}+\lambda_{c 2}+\mu_{b}} \\
\alpha_{3}=\frac{1}{\mu_{3}}
\end{gathered}
$$

and

$$
\alpha_{4}=\frac{\lambda_{c 1}}{\lambda_{c 1}+\mu_{c}}
$$

Under the following conditions, $\lambda_{a}=0.0001, \lambda_{b}=0.0002, \mu_{a}=0.0001$, $\mu_{b}=0.0002, \mu_{c}=0.0003, \mu_{3}=0.0004, \lambda_{c 1}=\lambda_{c 2}=\lambda_{c 3}=0.00001$, then $\alpha_{1}=0.2439024, \alpha_{2}=0.3921568, \alpha_{3}=2500, \alpha_{4}=0.032258,\left(\alpha_{1} / \lambda_{a}\right)=$ 2439.024, $\left(\alpha_{2} / \lambda_{b}\right)=1960.784,\left(\lambda_{b} / \mu_{3}\right)=0.5,\left(\lambda_{a} / \mu_{3}\right)=0.25,\left(\alpha_{4}\left(\lambda_{c 3}-\right.\right.$ $\left.\left.\lambda_{c 1}\right) / \lambda_{c 1}\right)=\left(\alpha_{4}\left(\lambda_{c 2}-\lambda_{c 1}\right) / \lambda_{c 1}\right)=0$ and $\left(\alpha_{4} / \lambda_{c 1}\right)=3225.8$ and Eqs. (8a) (8e) reduce to

$$
\begin{gathered}
P_{0}(t)=1-P_{1}(t)-P_{2}(t)-P_{3}(t)-P_{4}(t) \\
P_{1}(t)=0.2439024\left(1-P_{2}(t)-P_{3}(t)-P_{4}(t)\right)-2439.024 \frac{d P_{1}(t)}{d t} \\
P_{2}(t)=0.3921568\left(1-P_{1}(t)-P_{3}(t)-P_{4}(t)\right)-1960.784 \frac{d P_{2}(t)}{d t} \\
P_{3}(t)=0.5 P_{1}(t)+0.25 P_{2}(t)-2500.0 \frac{d P_{3}(t)}{d t}
\end{gathered}
$$


and

$$
P_{4}(t)=0.032258\left(1-P_{3}(t)\right)-3225.8 \frac{d P_{4}(t)}{d t}
$$

The equivalent circuit of Eqs. (9a)-(9e) is shown in Figure 5 and its SPICE input file is shown in Figure 6 where the initial conditions $P_{n}(0)=0, n=1,2,3,4$ are set to zero. The transient results of Figure 7 show the state probabilities $P_{n}(t)=0, n=0,1, \ldots, 4$ and the system reliability. Tables I-III show the steady-state system availability and MTTF for three different models of the system. Also shown in Tables I-III are the results obtained using the Laplace transform method [5]. From Tables I-III it can be seen that the results obtained using SPICE simulation are in excellent agreement with the results obtained using the Laplace transform method.

Example 2 Warm standby systems with common-cause failures and human errors [1].

Figure 8 shows the state transition diagram along with the corresponding failure rates and repair rates of the warm standby systems with common-cause failures and human errors. [1]. The state transition
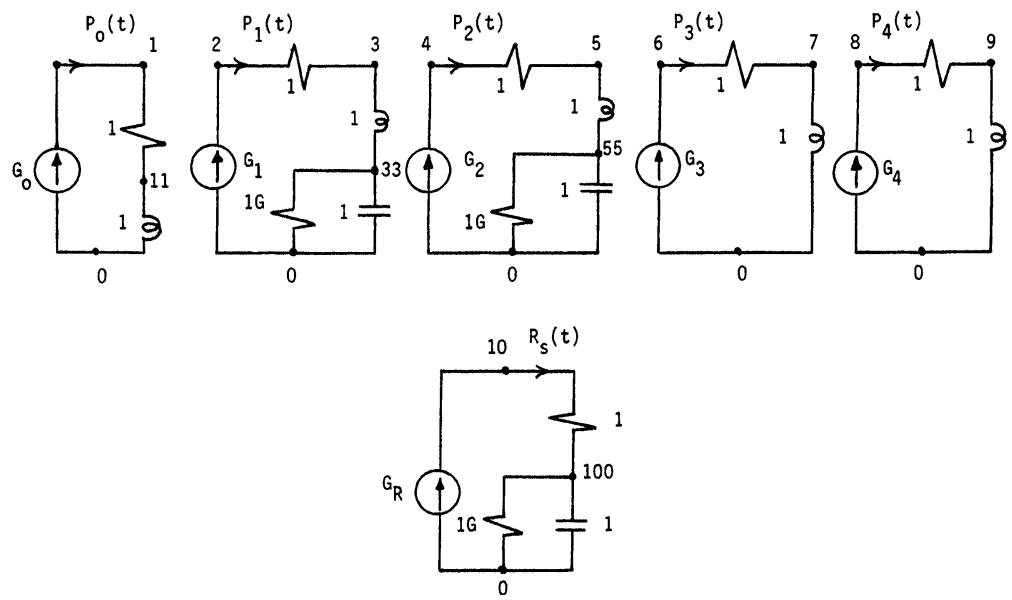

FIGURE 5 Equivalent circuit used for calculating the probabilities $P_{n}, n=0,1, \ldots, 4$ and the reliability $R_{s}$ for Example $1 V(100,0)=$ MTTF. 


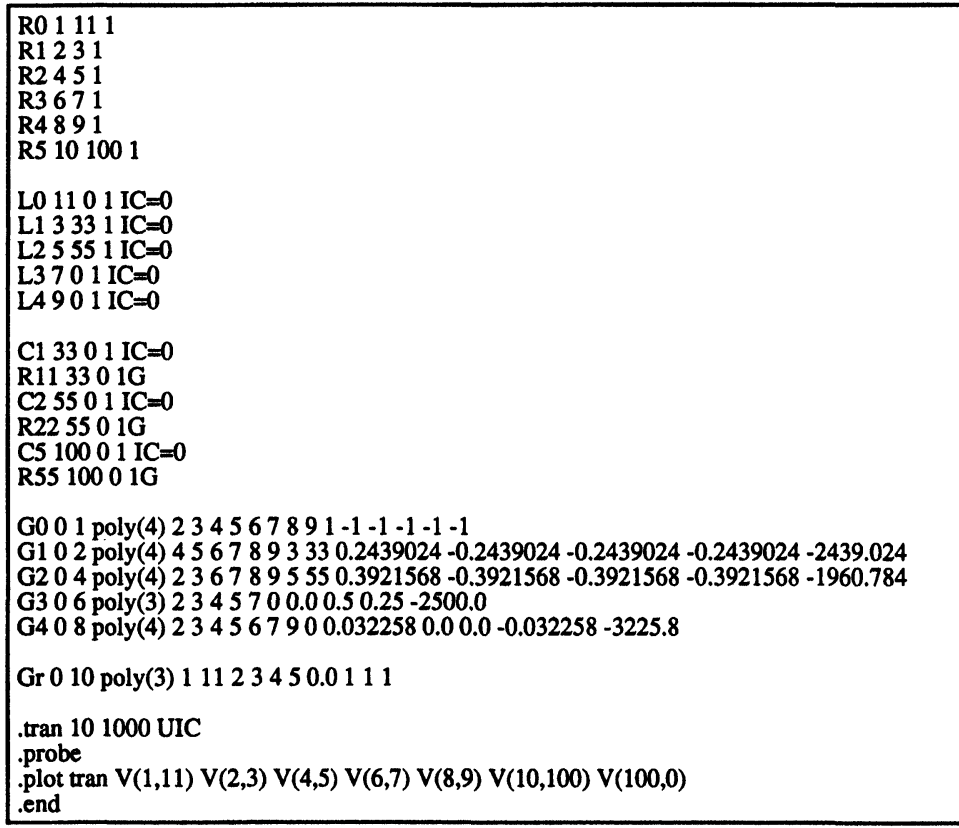

FIGURE 6 SPICE input file for the circuit of Figure 5 corresponding to the state transition diagram of Example 1.

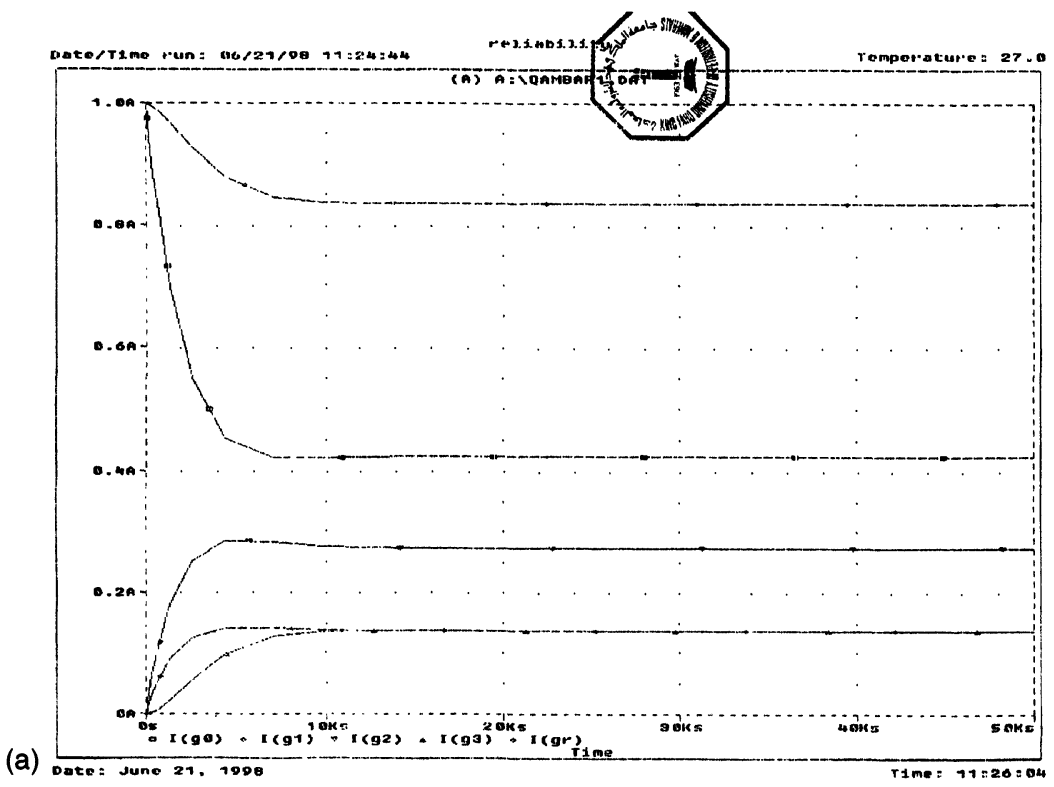

FIGURE 7 (a) Probabilities $P_{n}(t), n=0,1, \ldots, 4$; (b) system reliability $R_{s}(t)$ of the example of Figure 6 
(b)

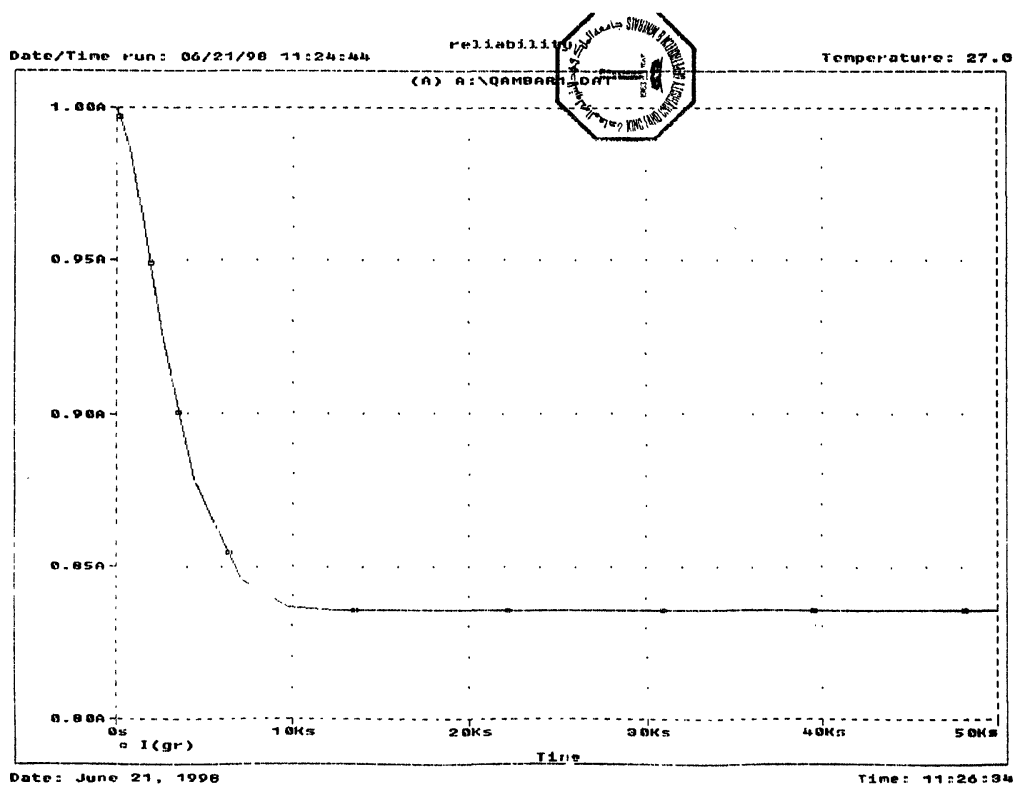

FIGURE 7 (Continued).

TABLE I Steady-state system availability of Model I of Example 1 with $\lambda_{a}=0.0001$, $\lambda_{b}=0.0002, \mu_{a}=0.0001, \mu_{b}=0.0002, \mu_{c}=0.0003, \mu_{3}=0.0004$

\begin{tabular}{lcc}
\hline & \multicolumn{2}{c}{$\begin{array}{c}\text { Steady state availability } \\
A V_{\text {ss }}\end{array}$} \\
\cline { 2 - 3 }$\lambda_{c 1}=\lambda_{c 2}=\lambda_{c 3}$ & SPICE & $\begin{array}{c}\text { Laplace } \\
\text { transform } \\
\end{array}$ \\
\hline simulation & method $[5]$ \\
\hline 0.0000 & & 0.8616 \\
0.00001 & 0.8571 & 0.8406 \\
0.00002 & 0.8352 & 0.8203 \\
\hline
\end{tabular}

TABLE II Steady-state system availability of Model II of Example 1 with $\lambda_{a}=0.0001$, $\lambda_{b}=0.0002, \mu_{a}=0.0001, \mu_{b}=0.0002, \mu_{c}=\mu_{3}=0.0$

\begin{tabular}{|c|c|c|}
\hline \multirow[b]{2}{*}{$\lambda_{c 1}$} & \multicolumn{2}{|c|}{$M T T F$} \\
\hline & $\begin{array}{c}\text { SPICE } \\
\text { simulation }\end{array}$ & $\begin{array}{c}\text { Laplace } \\
\text { transform } \\
\text { method }[5]\end{array}$ \\
\hline 0.0003 & 4615.0 & 4706.0 \\
\hline 0.0010 & 1764.0 & 1810.0 \\
\hline 0.0025 & 759.40 & 748.00 \\
\hline
\end{tabular}


TABLE III Steady-state system availability of Model III of Example 1 with $\lambda_{a}=0.0001$, $\lambda_{b}=0.0002, \mu_{a}=\mu_{b}=\mu_{c}=\mu_{3}=\lambda_{c 2}=\lambda_{c 3}=0.0$

\begin{tabular}{lcc}
\hline & \multicolumn{2}{c}{ MTTF } \\
\cline { 2 - 3 }$\lambda_{c 1}$ & $\begin{array}{c}\text { SPICE } \\
\text { simulation }\end{array}$ & $\begin{array}{c}\text { Laplace } \\
\text { transform } \\
\text { method [5] }\end{array}$ \\
\hline 0.0003 & 5833.0 & 5675.0 \\
0.0010 & 2692.0 & 2689.0 \\
0.0025 & 1250.0 & 1210.0 \\
\hline
\end{tabular}

$\mu$

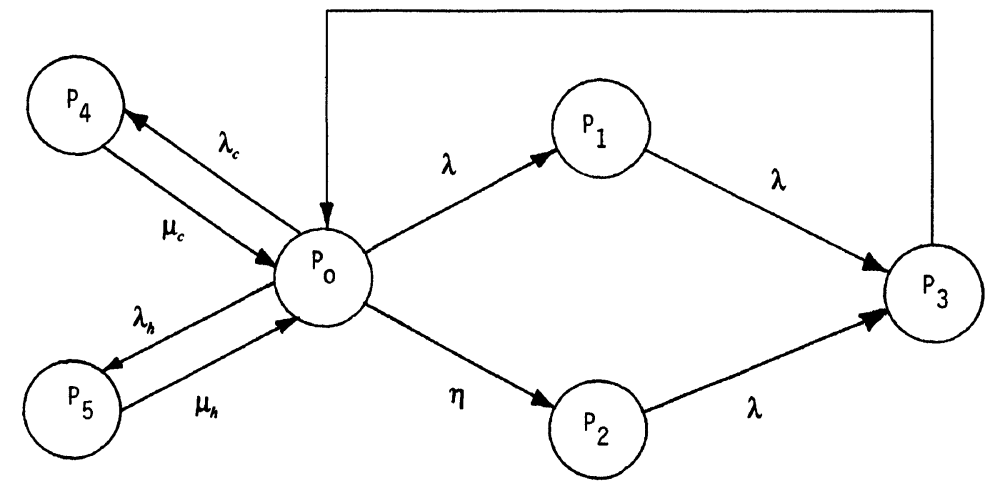

FIGURE 8 State transition diagram of Example 2 [1] State 0: One unit operating and standby operating, State 1: Operating unit failed and standby operating, State 2: Stand by unit failed in its standby mode, operating unit working, State 3: both units failed due to other than common-cause failure and human errors, State 4: both units failed due to common-cause failure, State 5: both units failed due to a human error.

equations of this system can be written as:

$$
\begin{gathered}
\frac{d P_{0}(t)}{d t}=-\left(\lambda+\lambda_{c}+\lambda_{h}+\eta\right) P_{0}(t)+\mu P_{3}(t)+\mu_{c} P_{4}(t)+\mu_{h} P_{5}(t) \\
\frac{d P_{1}(t)}{d t}=\lambda P_{0}(t)-\lambda P_{1}(t) \\
\frac{d P_{2}(t)}{d t}=\eta P_{0}(t)-\lambda P_{2}(t)
\end{gathered}
$$




$$
\begin{gathered}
\frac{d P_{3}(t)}{d t}=\lambda P_{1}(t)+\lambda P_{2}(t)-\mu P_{3}(t) \\
\frac{d P_{4}(t)}{d t}=\lambda_{c} P_{0}(t)-\mu_{c} P_{4}(t)
\end{gathered}
$$

and

$$
\frac{d P_{5}(t)}{d t}=\lambda_{h} P_{0}(t)-\mu_{h} P_{5}(t)
$$

Following the procedure described in the previous section, assuming that Eq. (9a) is the source equation, Eqs. $(9 a-9 e)$ can be rewritten as

$$
\begin{array}{r}
P_{0}(t)=1-P_{1}(t)-P_{2}(t)-P_{3}(t)-P_{4}(t)-P_{5}(t) \\
P_{1}(t)=\frac{1}{2}\left(1-P_{2}(t)-P_{3}(t)-P_{4}(t)-P_{5}(t)-\frac{1}{\lambda} \frac{d P_{1}(t)}{d t}\right) \\
P_{2}(t)=\alpha_{2}\left(1-P_{1}(t)-P_{3}(t)-P_{4}(t)-P_{5}(t)-\frac{1}{\eta} \frac{d P_{2}(t)}{d t}\right) \\
P_{3}(t)=\alpha_{3}\left(P_{1}(t)+P_{2}(t)-\frac{1}{\lambda} \frac{d P_{3}(t)}{d t}\right) \\
P_{4}(t)=\alpha_{4}\left(1-P_{1}(t)-P_{2}(t)-P_{3}(t)-P_{5}(t)-\frac{1}{\lambda_{c}} \frac{d P_{4}(t)}{d t}\right)
\end{array}
$$

and

$$
P_{5}(t)=\alpha_{5}\left(1-P_{1}(t)-P_{2}(t)-P_{3}(t)-P_{4}(t)-\frac{1}{\lambda_{h}} \frac{d P_{5}(t)}{d t}\right)
$$

where

$$
\alpha_{2}=\frac{\eta}{\lambda+\eta}
$$




$$
\begin{gathered}
\alpha_{3}=\frac{\lambda}{\mu} \\
\alpha_{4}=\frac{\lambda_{c}}{\lambda_{c}+\mu_{c}}
\end{gathered}
$$

and

$$
\alpha_{5}=\frac{\lambda_{h}}{\lambda_{h}+\mu_{h}}
$$

Under the following conditions $\mu_{1}=\mu_{2}=0, \lambda=0.0001, \lambda_{c}=0.00001$, $\eta=0.00005, \mu_{c}=0.001, \mu_{h}=0.002, \lambda_{h}=0.00003$ and $\mu=0.001$, Eqs. $(11 \mathrm{a}-11 \mathrm{f})$ can be rewritten as

$$
\begin{gathered}
P_{0}(t)=1-P_{1}(t)-P_{2}(t)-P_{3}(t)-P_{4}(t)-P_{5}(t) \quad(12 \mathrm{a}) \\
P_{1}(t)=0.5\left(1-P_{2}(t)-P_{3}(t)-P_{4}(t)-P_{5}(t)\right)-5000.0 \frac{d P_{1}(t)}{d t} \quad(12 \mathrm{~b}) \\
P_{2}(t)=0.33333333\left(1-P_{1}(t)-P_{3}(t)-P_{4}(t)-P_{5}(t)\right) \\
-6666.6666 \frac{d P_{2}(t)}{d t} \\
P_{3}(t)=0.1\left(P_{1}(t)+P_{2}(t)\right)-1000.0 \frac{d P_{3}(t)}{d t} \\
P_{4}(t)=0.0099009\left(1-P_{1}(t)-P_{2}(t)-P_{3}(t)-P_{5}(t)\right)-990.09 \frac{d P_{4}(t)}{d t}
\end{gathered}
$$

and

$$
P_{5}(t)=0.0147783\left(1-P_{1}(t)-P_{2}(t)-P_{3}(t)-P_{4}(t)\right)-492.61 \frac{d P_{5}(t)}{d t}
$$

The equivalent circuit of Eqs. (12a)-(12f) is shown in Figure 9 and its SPICE input file is shown in Figure 10 where the initial conditions $P_{n}(0)=0, n=1,2, \ldots, 5$ are set to zero. The transient results of Figure 11 show the state probabilities $P_{n}(t)=0, n=0,1, \ldots, 5$, and the system reliability as functions of time. Tables IV and V show the 


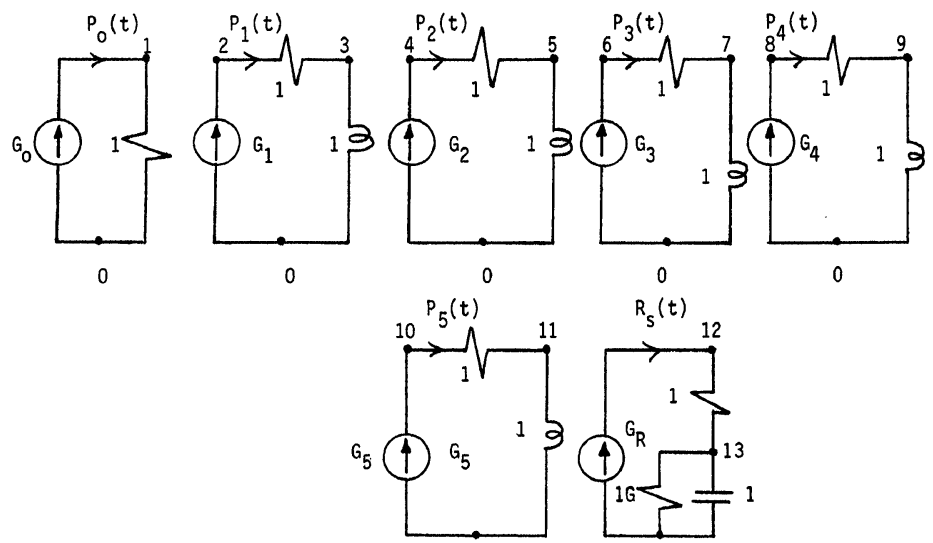

FIGURE 9 Equivalent circuit used for calculating the probabilities $P_{n}, n=0,1, \ldots, 5$ and the reliability $R_{s}$ for Example $2 . V(13,0)=$ MTTF.

\begin{tabular}{|c|}
\hline $\begin{array}{lllll}\text { R0 } & 1 & 0 & 1 \\
\text { R1 } & 2 & 3 & 1 \\
\text { R2 } & 4 & 5 & 1 \\
\text { R3 } & 6 & 7 & 1 \\
\text { R4 } & 8 & 9 & 1 \\
\text { R5 } & 10 & 11 & 1 \\
\text { R6 } & 12 & 13 & 1\end{array}$ \\
\hline 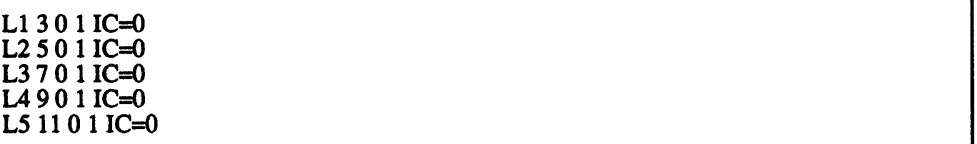 \\
\hline $\begin{array}{l}\text { C1 } 1301 \text { IC }=0 \\
R 71301 G\end{array}$ \\
\hline $\begin{array}{l}\text { G0 } 01 \text { poly(5) } 23445678910111-1-1-1-1-1 \\
\text { G1 } 02 \text { poly(5) } 45678991011300.5-0.5-0.5-0.5-0.5-5000.0 \\
\text { G2 } 04 \text { poly(5) } 236787991011500.333333-0.333333-0.333333-0.333333-0.333333 \\
\quad-6666.6666 \\
\text { G3 } 06 \text { poly(3) } 2345700.00 .10 .1-1000.0 \\
\text { G4 } 08 \text { poly(5) } 2345671011900.0099009-0.0099009-0.0099009-0.0099009-0.0099009 \\
\quad-990.09 \\
\text { G5 } 010 \text { poly(5) } 234567891100.0147783-0.0147783-0.0147783-0.0147783-0.0147783 \\
\quad-492.61\end{array}$ \\
\hline Gr $012 \operatorname{poly}(3) 1023450.0111$ \\
\hline $\begin{array}{l}\text {.tran } 10000150000 \text { UIC } \\
\text { probe } \\
\text { print tran } \mathrm{V}(1,0) \mathrm{V}(2,3) \mathrm{V}(4,5) \mathrm{V}(6,7) \mathrm{V}(8,9) \mathrm{V}(10,11) \mathrm{V}(12,13) \mathrm{V}(13,0) \\
\text {.end }\end{array}$ \\
\hline
\end{tabular}

FIGURE 10 SPICE input file of the circuit of Figure 9 corresponding to the state transition diagram of Example 2. 


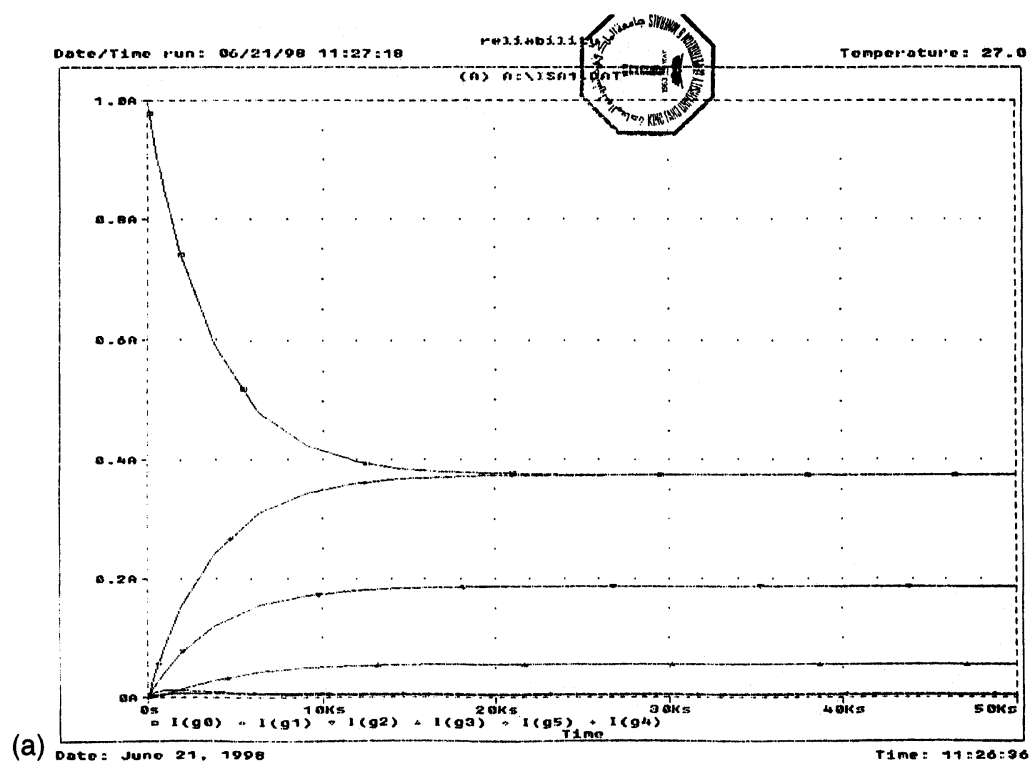

(a) Dato: Juno 21. 1998

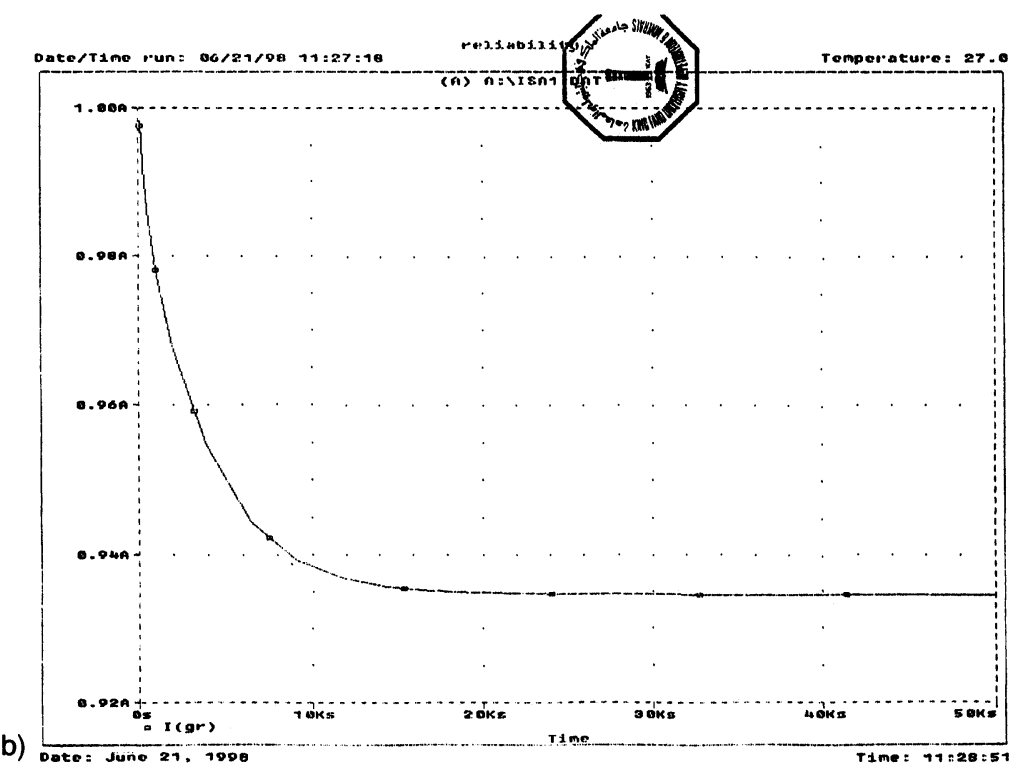

FIGURE 11 (a) Probabilities $P_{n}(t), n=0,1, \ldots, 5$; (b) system reliability $R_{s}(t)$ of the example of Figure 10. 
TABLE IV Steady-state system availability of Model I of Example 2 with $\lambda=0.0001$, $\lambda_{c}=0.00003, \eta=0.00005, \mu_{1}=\mu_{2}=0.0, \mu_{c}=0.001, \mu_{h}=0.002, \mu=0.001$

\begin{tabular}{lcc}
\hline & \multicolumn{2}{c}{ Steady-state availability } \\
$\lambda_{h}$ & \multicolumn{2}{c}{$A V_{\text {ss }}$} \\
\cline { 2 - 3 } & SPICE & Laplace \\
& simulation & transform \\
& & method [1] \\
\hline 0.0001 & 0.9158 & 0.9159 \\
0.0003 & 0.8834 & 0.8834 \\
0.0005 & 0.8532 & 0.8533 \\
\hline
\end{tabular}

TABLE V Steady-state system availability of Model II of Example 2 with $\lambda=0.0001$, $\lambda_{c}=0.00003, \eta=0.00005, \mu_{1}=\mu_{2}=0.0005, \mu_{c}=0.0001, \mu_{h}=0.0002, \mu=0.0003$

\begin{tabular}{lcc}
\hline & \multicolumn{2}{c}{ Steady-state availability } \\
$\lambda_{h}$ & \multicolumn{2}{c}{$A V_{\text {ss }}$} \\
\cline { 2 - 3 } & SPICE & Laplace \\
& simulation & method $[3]$ \\
\hline 0.00000 & & 0.7627 \\
0.00004 & 0.7653 & 0.6797 \\
0.00010 & 0.6818 & 0.5847 \\
\hline
\end{tabular}

steady-state system availability for two different models of the system. Also shown in Tables IV and V are the results obtained using the Laplace transform method [1]. From Tables IV and V it can be seen that the results obtained using SPICE simulation are in excellent agreement with the results obtained using the Laplace transform method.

\section{CONCLUSION}

It is evident, from the analysis presented and the examples considered in this paper, that SPICE circuit simulation program is an effective tool for calculating the time dependent probabilities, the system reliability, the system steady-state availability and the mean-time to failure of repairable multi-state systems described by Markov models. Especially for complicated systems where recourse to numerical solution of higher-order polynomial is inevitable, the use of SPICE is highly recommended as it saves time and avoids involvement in a sea of algebra. 


\section{References}

[1] Dhillon, B. S. and Yang, N. (1992). Reliability and availability of warm standby systems with common-cause failures and human errors, Microelectronics and Reliability, 32, 561-575.

[2] Dhillon, B. S. and Anude, O. C. (1993). Common-cause failure analysis of redundant system with non-repairable units, Microelectronics and Reliability, 33, $1499-1509$.

[3] Tan, Z. B. (1997). Reliability and availability analysis of two-unit warm standby microcomputer systems with self-reset function and repair facility, Microelectronics and Reliability, 37, 1251-1253.

[4] Dhillon, B. S. and Yang, N. (1997). Formulas for analyzing a redundant robot configuration with a built-in safety system, Microelectronics and Reliability, 37, $557-563$.

[5] Dhillon, B. S. and Viswanath, H. C. (1991). Reliability analysis of a non-identical unit parallel system with common-cause failures, Microelectronics and Reliability, 31, 429-441.

[6] Dhillon, B. S. and Yang, N. (1996). Availability analysis of a robot with safety system, Microelectronics and Reliability, 36, 169-177.

[7] Qamber, I. S. (1993). Flow graph development method, Microelectronics and Reliability, 33, 1387-1395.

[8] Abuelma'atti, M. T. and Qamber, I. S. (1997). SPICE application in the study of the behaviour of multi-state systems described by Markov models, Microelectronics and Reliability, 37, 609-613. 

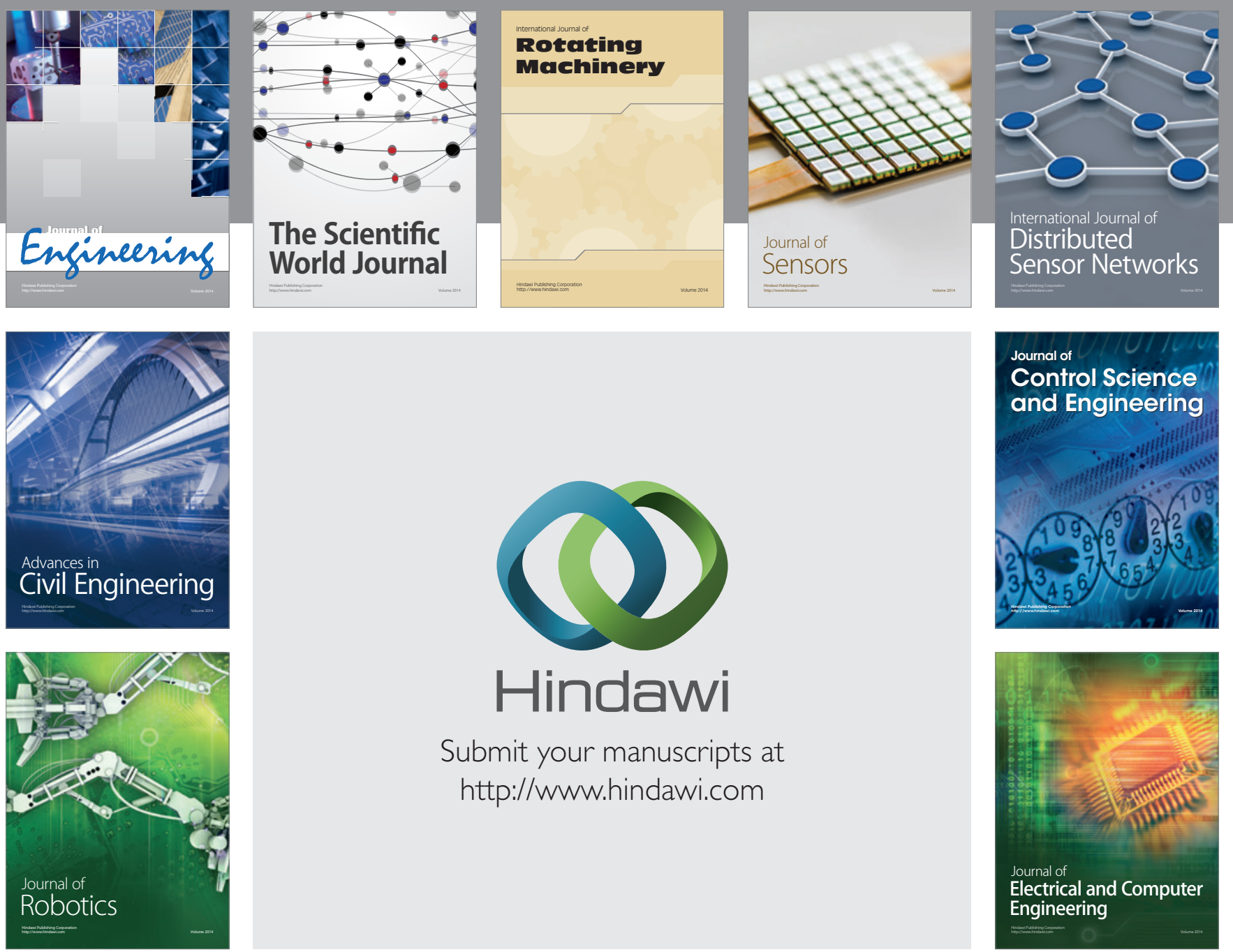

Submit your manuscripts at

http://www.hindawi.com
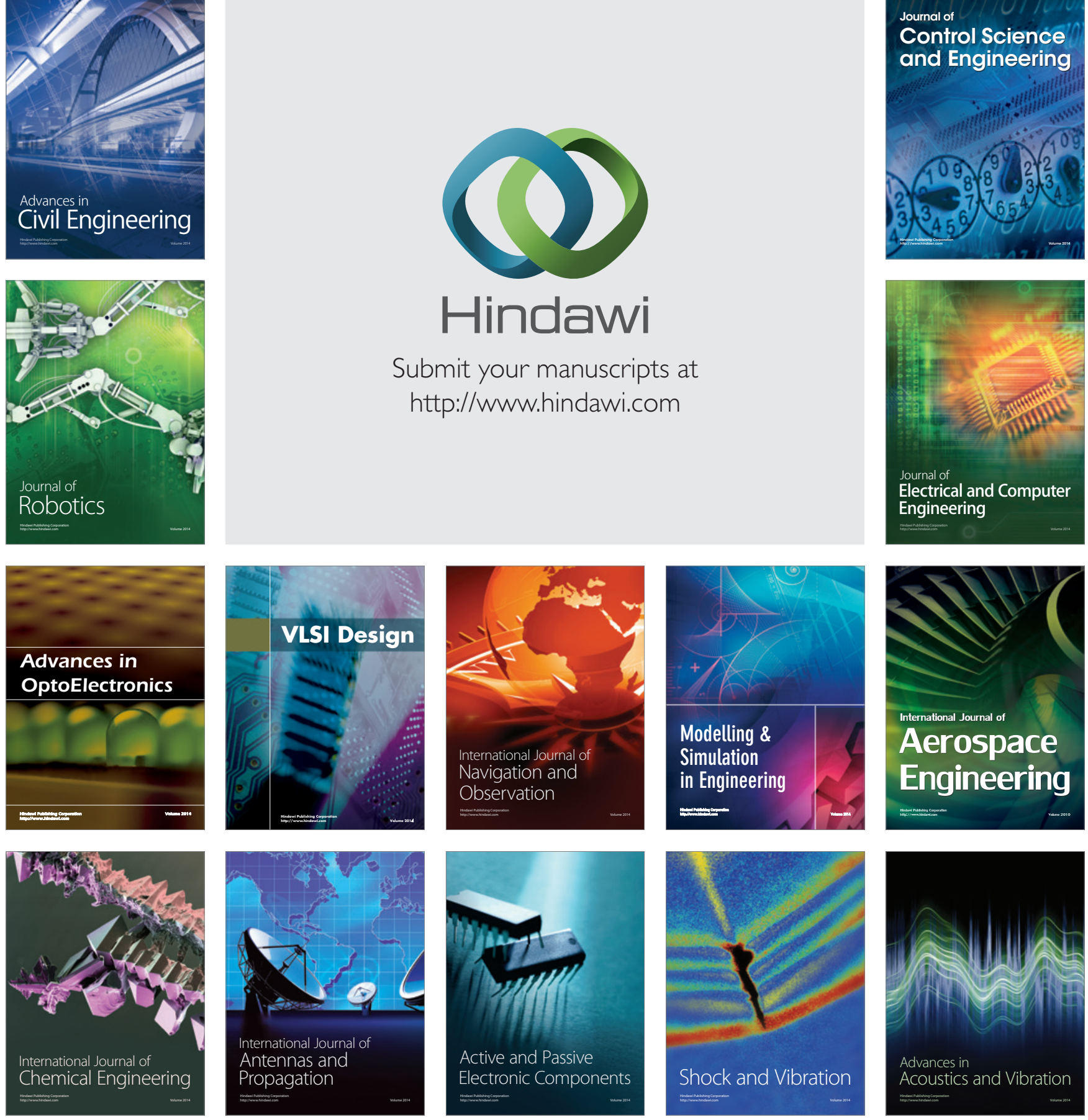